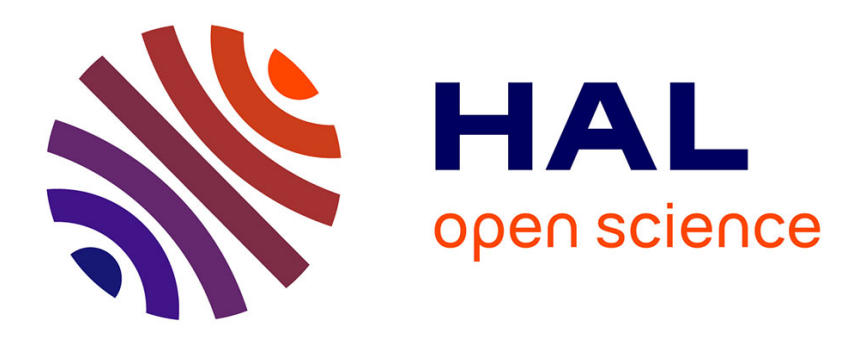

\title{
Parameterisations of interior properties of rocky planets
}

\author{
Lena Noack, Marine Lasbleis
}

\section{To cite this version:}

Lena Noack, Marine Lasbleis. Parameterisations of interior properties of rocky planets: An investigation of planets with Earth-like compositions but variable iron content. Astronomy and Astrophysics - A\&A, 2020, 638, pp.A129. 10.1051/0004-6361/202037723 . hal-02995481

\section{HAL Id: hal-02995481 \\ https://hal.science/hal-02995481}

Submitted on 9 Nov 2020

HAL is a multi-disciplinary open access archive for the deposit and dissemination of scientific research documents, whether they are published or not. The documents may come from teaching and research institutions in France or abroad, or from public or private research centers.
L'archive ouverte pluridisciplinaire HAL, est destinée au dépôt et à la diffusion de documents scientifiques de niveau recherche, publiés ou non, émanant des établissements d'enseignement et de recherche français ou étrangers, des laboratoires publics ou privés. 


\title{
Parameterisations of interior properties of rocky planets
}

\section{An investigation of planets with Earth-like compositions but variable iron content}

\author{
Lena Noack ${ }^{1}$ and Marine Lasbleis ${ }^{2,3}$ \\ 1 Department of Earth Sciences, Freie Universität Berlin, Malteserstr. 74-100, 12249 Berlin, Germany \\ e-mail: lena.noack@fu-berlin.de \\ 2 Laboratoire de Planétologie et Géodynamique, LPG, UMR 6112, CNRS, Université de Nantes, Université d'Angers, Nantes, France \\ e-mail: marine. lasbleis@univ-nantes.fr \\ 3 Earth-Life Science Institute, Tokyo Institute of Technology, Meguro, Tokyo 152-8551, Japan
}

Received 13 February 2020 / Accepted 28 April 2020

\begin{abstract}
Context. Observations of Earth-sized exoplanets are mostly limited to information on their masses and radii. Simple mass-radius relationships have been developed for scaled-up versions of Earth or other planetary bodies such as Mercury and Ganymede, as well as for one-material spheres made of pure water(-ice), silicates, or iron. However, they do not allow a thorough investigation of composition influences and thermal state on a planet's interior structure and properties.

Aims. In this work, we investigate the structure of a rocky planet shortly after formation and at later stages of thermal evolution assuming the planet is differentiated into a metal core and a rocky mantle (consisting of Earth-like minerals, but with a variable iron content).

Methods. We derived possible initial temperature profiles after the accretion and magma ocean solidification. We then developed parameterisations for the thermodynamic properties inside the core depending on planet mass, composition, and thermal state.

Results. We provide the community with robust scaling laws for the interior structure, temperature profiles, and core- and mantleaveraged thermodynamic properties for planets composed of Earth's main minerals but with variable compositions of iron and silicates.

Conclusions. The scaling laws make it possible to investigate variations in thermodynamic properties for different interior thermal states in a multitude of applications such as deriving mass-radius scaling laws or estimating magnetic field evolution and core crystallisation for rocky exoplanets.
\end{abstract}

Key words. equation of state - Earth - planets and satellites: interiors - planets and satellites: terrestrial planets

\section{Introduction}

Amongst the large rocky bodies in our Solar System, most of them present evidence of a past and/or present magnetic field (Stevenson et al. 1983), with the exception of Venus, for which no significant intrinsic magnetic field has been detected and no record of a past global magnetic field is available (Zhang et al. 2016). Planetary magnetic fields are generated by the dynamo effect in large volumes of electrically conducting liquid. For rocky bodies of sizes similar to the Earth, such a dynamo effect is supposed to happen in the liquid core and be driven by thermochemical convection (Stevenson et al. 1983). The existence of a magnetic field in the history of a planet is thus considered as strong evidence for past or current internal dynamics and the existence of a liquid core. The internal structure of a rocky body is difficult to assess. The Earth is the only planet for which the coverage in seismic stations and sources is good enough to provide us with a detailed structure of its interior. The Earth is thus used as an example, test case, and sometimes a law for structure in rocky bodies. Seismometers have been installed on two other bodies so far: the Moon and Mars. However, data points are sparse and can only give a first, rough constraint on the interior structure and interior properties. Observations of different rotational and orbital properties such as librations, Love numbers, and the moment of inertia of a planetary body also provide information on its global internal structure, such as the ice shell thickness for water-rich, icy bodies; the existence of a liquid core or subsurface liquid water ocean; or the concentration of mass in the centre (e.g. Thomas et al. 2016; Verhoeven et al. 2005).

For an exoplanet, obtaining its internal structure with enough confidence to discuss its internal state and dynamics is a very different challenge. The observations of Earth-sized exoplanets are mostly limited to information on their masses and radii. Simple mass-radius relationships have been developed for scaledup versions of Earth or other planetary bodies such as Mercury and Ganymede, or for one-material spheres made of pure water (-ice), silicates, or iron (e.g. Valencia et al. 2006; Wagner et al. 2011; Noack et al. 2016). In addition, constraints from the stellar composition and possible condensation and accretion scenarios can be used to constrain the interior composition of (sub-)Neptunian planets (Grasset et al. 2009; Rogers \& Seager 2010) as well as, more recently, low-mass rocky planets (Dorn et al. 2015; Unterborn \& Panero 2017).

For the long-term evolution of a rocky planet, the interior structure (such as core size), as well as the initial temperature profile have been identified as important parameters (Noack et al. 2014; Dorn et al. 2018). However, even for the 
planets in the Solar System, the initial energy budget and temperature profile in the first hundreds of Myr of thermal evolution are not constrained. During planet accretion, large amounts of the interior will be molten due to giant impacts, leading to a magma ocean (e.g. Nakajima \& Stevenson 2015). Depending on planet mass and time of accretion, the magma ocean may comprise the entire planet. Iron droplets segregate though the molten rock and concentrate at the bottom of the magma ocean and slowly sink towards the gravitational centre of the planet, leading to a release of additional gravitational energy to the mantle or core.

Once the surface temperature allows for efficient cooling in the upper part of the planet (due to erosion of the atmosphere), the magma ocean can efficiently cool in the parts where the liquid melt dominates convection. It is typically assumed that the magma ocean will freeze from the bottom up (Abe 1997; Labrosse et al. 2007), though different possible freezing scenarios (such as floating of solids in melt or freezing starting at intermediate depths, Nomura et al. 2011) are still under debate. The heat flow from the core into the mantle is thus reduced, since solid layers transport heat much less efficiently than liquid layers. While the magma ocean freezes out, solidstate convection would be assumed to take place in the silicate mantle (Ballmer et al. 2017; Maurice et al. 2017; Boukaré et al. 2018), leading to an adiabatic temperature profile in the final, entirely solid mantle. As a consequence of this assumed evolution of planet accretion and magma ocean solidification, Stixrude (2014) predicted a possible initial temperature profile for planets of Earth-like composition with varying planet mass based on the solidus melting temperatures of iron and silicates. We expanded this idea by investigating different temperature profiles including the predicted profiles from Stixrude (2014) as intermediate scenario. To obtain an estimate for the maximal temperature of iron during core formation, one may therefore assume that the core could have formed while the entire mantle was still molten. An even hotter core would be expected to cool very fast due to efficient heat transport through the magma ocean until the bottom of the mantle solidified. One can therefore assume that the maximal initial temperature of the uppermost layer of the metal core would correspond to the liquidus temperature of the mantle at the end of core formation.

In published exoplanet evolution studies, different initial thermal profiles after the magma ocean stage were already proposed, using adiabatic profiles with different uppermantle potential temperatures and assuming different temperature jumps at the core-mantle boundary (Valencia et al. 2006; Papuc \& Davies 2008; Tackley et al. 2013; Stamenković et al. 2012; Noack \& Breuer 2014). Much lower temperatures at the core-mantle boundary were typically chosen than those predicted by Stixrude (2014).

Especially for planets more massive than Earth (superEarths), the initial temperature is essential in determining the possible evolution scenarios of the interior, surface, and atmosphere of these planets (Stamenković et al. 2012; Stamenković \& Breuer 2014; Noack \& Breuer 2014; O’Neill et al. 2016), since both volcanic activity and plate tectonics are related to the interior convective behaviour and stresses (Stein et al. 2004; Van Heck \& Tackley 2008; Crowley \& O'Connell 2012). Massive planets may exhibit a stiffer rheology in their deep interiors (Stamenković et al. 2011, 2012), which for high temperatures in the lower mantle can evolve into a self-regulating state (Tackley et al. 2013). The initial profile may determine which convective state is favoured in the deep mantle of these super-Earths.
The temperature profile is therefore crucial for setting the later thermal evolution of rocky planets, including whether or not they can transition into a plate tectonics regime (Stein et al. 2004; Sandu et al. 2011; Noack \& Breuer 2014; Stamenković \& Breuer 2014). The adiabatic profile, as well as the convective behaviour, strongly depend on the local thermodynamic (e.g. density, heat capacity, thermal expansion coefficient) and transport properties (such as thermal conductivity and viscosity). However, to obtain these local material properties, an interior structure model would first be needed to understand the interplay between temperature, pressure, mineral phases, and degree of differentiation. Such a model would need to be coupled to a Gibbs minimisation code solving for single mineral stability regions and thermodynamic properties based on equation of states (e.g. Connolly 2009; Stixrude \& Lithgow-Bertelloni 2011), or use lookup tables for common mantle minerals derived from such codes to save computational speed. In any case, developing and employing such a sophisticated code to obtain realistic, composition- and pressure-dependent profiles goes beyond the scope of most modern studies that seek to obtain first-order ideas of rocky planets' interior properties, core information, and evolution models.

We therefore derived parameterisations of interior structure and thermodynamic properties, as well as different temperature profile estimates as needed for common investigations of rocky exoplanets composed of Earth-like minerals (but variable planet compositions in terms of iron content), to be used in the exoplanet community. We constructed two end-member temperature profiles for a hot and a cold core state (reflecting an initial or late evolution stage) by either considering or neglecting a possible temperature jump at the core mantle boundary (CMB) and by calculating the extent of this temperature jump depending on composition, interior structure, and planet mass. We then show the effect that these two end-member temperature profiles have on planetary parameters and, especially, core thermodynamic parameters.

\section{Methods}

Internal structures of planets were calculated to determine pressure, temperature, and other material properties profiles. We explored planetary masses from 0.8 to 2 Earth masses, considering variations of compositions leading to different core sizes. Following Stixrude (2014), we considered the thermal profile of the core after the full crystallisation of the silicates at the CMB, which can be used as an initial temperature profile for long-term evolution investigations of rocky planets.

\subsection{Melting and adiabatic temperature profiles}

Here, we predict possible initial temperature profiles in the mantle and core once the mantle is in the solid state, based on the work of Stixrude (2014). In that study, it was assumed that the initial temperature profile upon magma ocean solidification would be adiabatic in the mantle, starting from the solidus melting temperature in the upper mantle (set in our study at a depth of $50 \mathrm{~km}$ ), and that the temperature at the CMB would reflect the bottom mantle solidus melting temperature. In other words, once the bottom of the magma ocean starts to freeze, no further heat loss from the core is considered. This case is labelled as the "warm" case in Fig. 1. If the core efficiently cools over longer timescales, or if heat can be lost during the magma ocean solidification from core to mantle without any restrictions, the lowest expected temperature at the $\mathrm{CMB}$ would be equal to the 

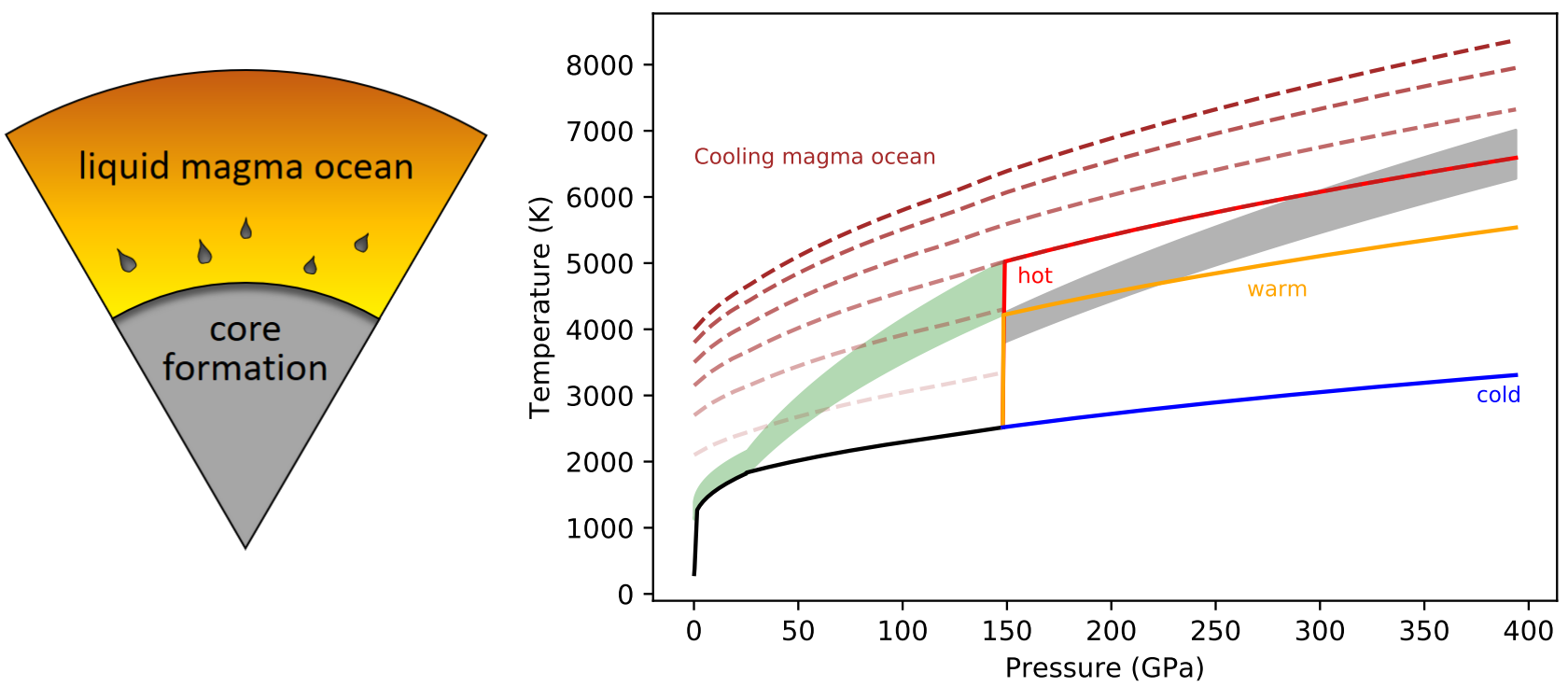

Fig. 1. Sketch of the evolution of temperature during the magma ocean and core formation stage. Brown, dashed curves depict the cooling temperature profile with time; for simplicity, we assume a super-heated interior to an extent that the entire interior would initially be molten. The green and grey areas show the melting region (from solidus to liquidus melting temperature) for the mantle and the core, respectively, following Stixrude (2014). The assumed final temperature profile of the mantle after magma ocean solidification is shown in black, starting at the solidus temperature below the surface thermal boundary layer and increasing adiabatically with depth throughout the rocky mantle. For the core, three possible temperature scenarios are shown, set at the CMB, either to the mantle liquidus temperature (hot, red), the mantle solidus temperature (warm, orange) or the adiabatic mantle temperature (cold case, blue). While the first two cases (hot and warm) resemble possible temperatures during and at the end of the magma ocean stage, the cold scenario mimics an old planet that experienced efficient cooling.

bottom, adiabatic mantle temperature. We also investigated this "cold" end-member scenario in our study. We also considered a second end-member scenario with a super-heated core by setting the core surface temperature to the mantle liquidus temperature at that pressure (labelled as the "hot" case in Fig. 1). Here, we did not consider any temperature variations in the mantle, which can also have a small effect on the mantle thermodynamic parameters. An adiabatic profile will only occur for a strongly convecting mantle. For weakly convecting areas (e.g. the lower mantle for massive planets, Stamenković et al. 2012), the temperatures may be higher than assumed here. However, Tackley et al. (2013) suggested that a stiffer lower mantle would heat up until strong convection (and hence cooling of the lower mantle) would initiate again, therefore again leading to a temperature profile close to an adiabatic profile. The main effect of a warmer mantle on our parameterisations would be a reduced temperature contrast at the CMB. In this study, however, we concentrated only on the temperature effect for core properties.

In Stixrude (2014), only Earth-like mantle and core fractions were considered, leading to scaled-up models of Earth with larger masses. The variability with iron content and mantle composition was not investigated in this pioneering study, but is treated in the present study.

Following Dorn et al. (2018), we used the following solidus and liquidus melting temperatures (calculated in $\mathrm{K}$ ) for pressures below $17 \mathrm{GPa}$,

$$
\begin{aligned}
T_{\text {melt,sol }}= & 1409.15+134.2 \cdot p-6.581 \cdot p^{2}+0.1054 \cdot p^{3} \\
& +\left(102.0+64.1 \cdot p-3.62 \cdot p^{2}\right) \cdot\left(0.1-\# \mathrm{Fe}_{\mathrm{M}}\right) \\
T_{\text {melt,liq }}= & 2035.15+57.46 \cdot p-3.487 \cdot p^{2}+0.0769 \cdot p^{3},
\end{aligned}
$$

where pressure $p$ is given in $\mathrm{GPa}$, and where $\# \mathrm{Fe}_{\mathrm{M}}$ is the iron number of the mantle defined as the ratio of iron-bearing components $\left(\mathrm{FeO}, \mathrm{FeSiO}_{3}\right.$ and $\left.\mathrm{Fe}_{2} \mathrm{SiO}_{4}\right)$ over magnesium-rich minerals $\left(\mathrm{MgO}, \mathrm{MgSiO}_{3}\right.$ and $\left.\mathrm{Mg}_{2} \mathrm{SiO}_{4}\right)$ and lies between 0 and 1 .
For higher pressures, we used the melting formulation from Stixrude (2014):

$T_{\text {melt }}=5400 \cdot(p / 140)^{0.48} /\left(1-\ln \left(1-\# \mathrm{Fe}_{\mathrm{M}}-X_{\mathrm{M} 0}\right)\right)$,

where $X_{\mathrm{M} 0}$ denotes the difference in melting temperature from liquidus to solidus (hence it is set to zero for the hot profile to obtain the liquidus temperature) and $\# \mathrm{Fe}_{\mathrm{M}}$ gives a further reduction of the melting temperature accounting for the influence of iron (similarly to iron influence in the upper mantle, Dorn et al. 2018). For the core, again following Stixrude (2014), we use

$T_{\text {melt }}=6500 \cdot(p / 340)^{0.515} /\left(1-\ln \left(1-X_{\mathrm{S}}\right)\right)$.

$X_{\mathrm{S}}$ is the mole fraction of lighter elements in the core, reducing the melting temperature accordingly. In our study, we assumed a pure iron core, hence $X_{\mathrm{S}}=0$.

\subsection{Interior structure depending on planet mass and composition}

To determine the adiabatic temperature profile and thermodynamic parameters such as the density and thermal expansivity in mantle and core, we made use of an interior structure model (Noack et al. 2017) solving the hydrostatic, Poisson, and mass equations. In the model, the planet mass and compositional information were used as inputs, and the interior properties and radii of the core and planet were determined self-consistently. The mantle mineral species in the model are restricted to the EoS (equation of state) database in Stixrude \& Lithgow-Bertelloni (2011) using a third-order Birch-Murnaghan equation of state. For a first-order approximation of thermodynamic properties, here we only focus on the rock-forming elements $\mathrm{Mg}, \mathrm{Si}$, $\mathrm{O}$, and $\mathrm{Fe}$, which make up about $95 \%$ of Earth's mantle (McDonough \& Sun 1995), leading to the here investigated mineral phases: olivine, wadsleyite, ringwoodite, bridgmanite, magnesiowüstite, and post-perovskite (Van Hoolst et al. 2019). The 

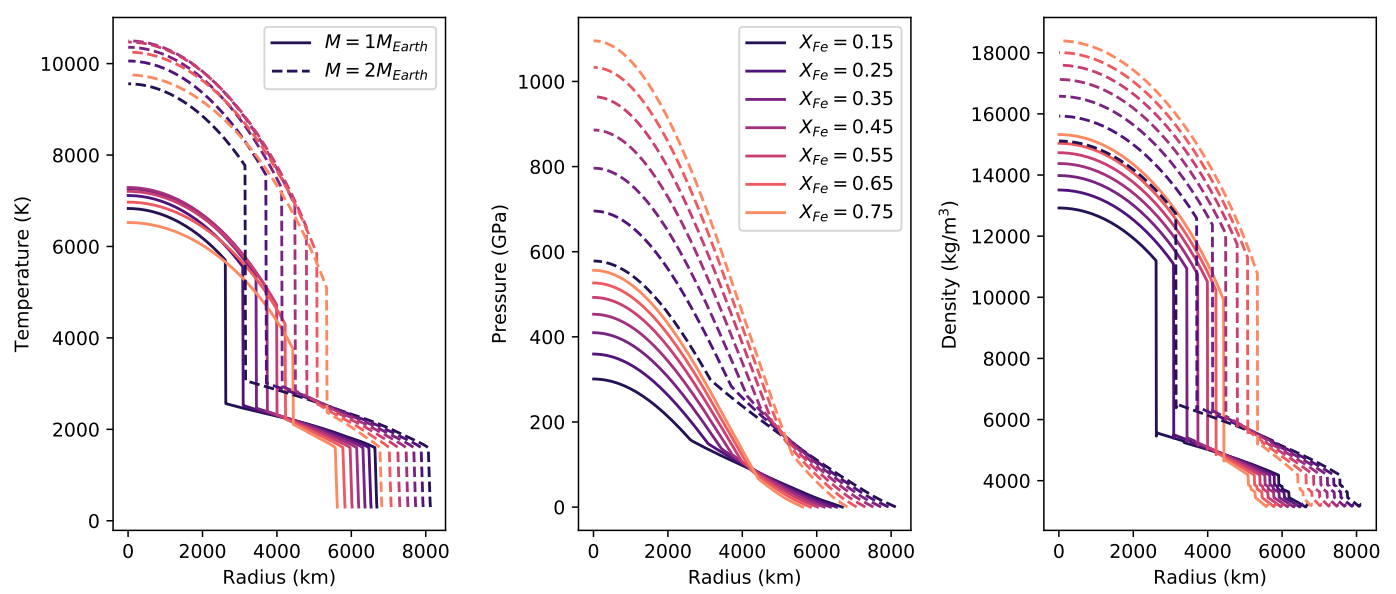

Fig. 2. Temperature (left), pressure (centre), and density (right) profiles for two example planet masses of one and two Earth masses considering the hot temperature scenario and assuming different planet iron contents.

mantle consists of different mixtures of $\left(\mathrm{Mg}_{1-\# \mathrm{Fe}_{\mathrm{M}}}, \mathrm{Fe}_{\mathrm{HFe}_{\mathrm{M}}}\right) \mathrm{O}$ and $\mathrm{SiO}_{2}$. For the core, we used pure iron and applied the Holzapfel equation of state from Bouchet et al. (2013).

The choice of the EoS applied to mantle and core can have an influence on the interior structure profiles, and hence our parameterisations. Especially for planets with masses well above one Earth mass, laboratory data is scarce at best, and the EoS need to be extrapolated well beyond the range of their validity. Baumeister et al. (2018) and Wagner et al. (2011) showed that the choice of mantle and core EoS on the interior structure has a rather small influence. Hakim et al. (2018) compared several different iron equations of state from the literature together with a new ab initio law derived for high pressures, and could show that the commonly used EoS start to strongly deviate from each other for pressures above $\sim 1 \mathrm{TPa}$. In that study, the Holzapfel EoS (Bouchet et al. 2013) could be confirmed by the new ab initio model, which is why we selected this EoS to model our core state. We limited our mass range to two Earth masses, to be in the confidence interval of the EoS selected for this study.

To constrain the initial temperature profile after magma ocean solidification, we used the derived thermodynamic properties in the mantle and core to obtain an adiabatic profile, starting from the melting temperature below an initially assumed lithosphere of $50 \mathrm{~km}$, which is approximately $1600 \mathrm{~K}$ (depending on mass and composition).

\section{Results}

We derived new scaling laws and first-principles relationships for main planetary parameters such as the planet and core radii, thermal expansion coefficient for mantle and core for different temperature profiles, and the expected temperature jump at the core-mantle boundary for the hot end-member temperature profile from Sect. 2.1 for rocky planets with an Earth-like mineralogy. For thermodynamic or planetary parameters that are strongly temperature dependent, we included a temperature dependence in the parameterisations. We used a mix of analytically derived equations based on first-order physical principles combined with empirical scaling laws derived via a regression analysis to find correlations between different parameters and to formulate simple, reproducible scaling laws.

For that purpose, we first used our interior structure model to investigate the interior structure and thermal state for planets with masses between 0.8 and 2 Earth masses (where the equations of state that we use are still applicable), planet iron mass fractions between 0.15 and 0.8 , and with variable iron contents in the mantle with a mass fraction up to 0.15 , which can be expressed via the mantle iron number $\# \mathrm{Fe}_{\mathrm{M}}$, and which we varied between 0 and 0.2 . In this study, the mantle iron number refers to the different mineral fractions of $(\mathrm{Mg}, \mathrm{Fe})_{2} \mathrm{SiO}_{4}$ and their higher pressure mineral equivalents. The resulting temperature, pressure, and density profiles for several hot end-member cases are given in Fig. 2.

In Fig. 3, we compare the cold and hot end-member cases for model cases for one and two Earth masses, an iron content of 0.35 , and a mantle iron number $\# \mathrm{Fe}_{\mathrm{M}}=0.1$ (this configuration best resembles Earth's interior structure). The main influence of the temperature profile is on the thermal expansion coefficient and heat capacity of the core (since the mantle temperature does not vary in our study). A weak influence of temperature can be observed for the density, though the influence on planet radius is negligible (see discussion). Furthermore, we compared our density profile with the preliminary reference Earth model (PREM500, Durek \& Ekström 1996). A slight variation can be observed in the core density, since we neglected core solidification as well as light elements in the core. Our core density is therefore slightly higher than in the PREM model, leading to a smaller core radius. On the other hand, our mantle density profile closely resembles the PREM model. In the temperature plot, the predicted initial temperature profile based on Valencia et al. (2006) and Stixrude (2014), as well as the initial core and upper mantle temperatures based on Papuc \& Davies (2008), were added for comparison. Our cold temperature profile is below the literature values, since it resembles a later stage in planet evolution. Our hot end-member scenario, on the other hand, exceeds predicted values from literature by several thousand $\mathrm{K}$. The temperature profile suggested by Stixrude (2014) (coinciding with our warm scenario) lies in-between our end-member curves but is larger than the other literature values. Initial temperature values used in other studies, such as Noack \& Breuer (2014), Tackley et al. (2013), are in general comparable to the values by Valencia et al. (2006) and Papuc \& Davies (2008). If our temperature scaling based on Stixrude (2014) is correct, then initial temperatures have so far been severely underestimated in studies investigating the thermal evolution of rocky exoplanets (Papuc \& Davies 2008; Valencia et al. 2007; Tackley et al. 2013; Noack \& Breuer 2014; Noack et al. 2017; Dorn et al. 2018). On the other hand, one could argue that some planets may transition quickly into a plate tectonics regime, where the mantle would be expected to cool much faster than for a stagnant-lid scenario. 

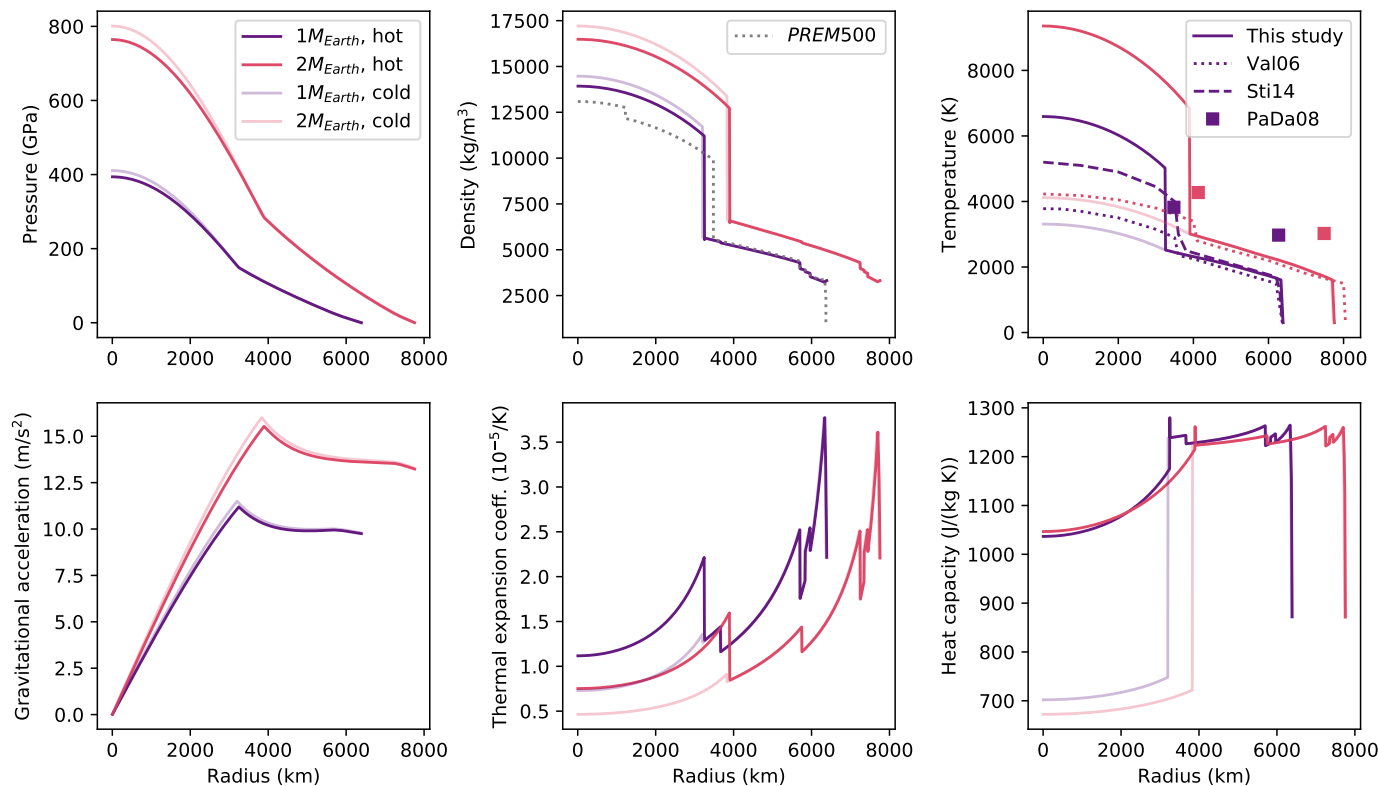

Fig. 3. Comparison of interior structure and thermodynamic parameter profiles for one and two Earth masses for an Earth-like composition for both the hot and the cold end-member temperature cases. In addition, we list reference profiles for density and temperature from the literature (PREM500: Durek \& Ekström 1996, Val06: Valencia et al. 2006, Sti14: Stixrude 2014, PaDa08: Papuc \& Davies 2008). Further explanation is given in the text.

In that case, mantle temperatures as assumed in some of these studies would be reached within a short time frame.

Below we list our derived scaling laws for all main planetary parameters. We added the average error plus/minus the standard deviation from the values giving a $68 \%(1 \sigma)$ confidence interval. Error refers here to the difference between parameter values determined by the set of equations compared to the actual values derived from the interior structure model.

\subsection{Planet radius}

The planet radius varies mostly with planet mass and planet iron content, where the distribution of iron between mantle and core and the interior temperature play only a minor role (see discussion section). We derived the following empirical scaling law based on our interior structure model results, where $X_{\mathrm{Fe}}$ is the weight iron fraction of the planet (from 0.15 to 0.8 ) and $M_{\text {Earth }}=5.972 \times 10^{24}$ :

$R_{\mathrm{p}}[\mathrm{km}]=\left(7030-1840 \cdot X_{\mathrm{Fe}}\right)\left(\frac{M_{\mathrm{p}}}{M_{\text {Earth }}}\right)^{0.282}$.

The scaling law compares well with similarly derived scaling laws in the literature (Valencia et al. 2006; Wagner et al. 2011; Noack et al. 2016); the exponent (0.282) lies within the literature range $(0.26-0.3)$.

\subsection{Core mass fraction}

Before we can determine the radius of the core, we need to know the core mass fraction $X_{\mathrm{CMF}}$, which gives the fraction of iron that is not taken up in mantle minerals and accumulated in the centre of the planet. The iron mass fraction in the mantle $X_{\mathrm{FeM}}$ is

$X_{\mathrm{FeM}}=\frac{2 \# \mathrm{Fe}_{\mathrm{M}} M_{\mathrm{Fe}}}{2\left(\left(1-\# \mathrm{Fe}_{\mathrm{M}}\right) M_{\mathrm{Mg}}+\# \mathrm{Fe}_{\mathrm{M}} M_{\mathrm{Fe}}\right)+M_{\mathrm{Si}}+4 M_{\mathrm{O}}}$, for molar masses $m_{\mathrm{Fe}}=55.845, m_{\mathrm{Mg}}=24.305, m_{\mathrm{Si}}=28.0855$ and $m_{\mathrm{O}}=15.999 \mathrm{~g} \mathrm{~mol}^{-1}$. The calculation is based on our assumption that the mantle is composed of mixtures of $\mathrm{Mg}_{2} \mathrm{SiO}_{4}$ and $\mathrm{Fe}_{2} \mathrm{SiO}_{4}$ (and according higher pressure minerals), where $\# \mathrm{Fe}_{\mathrm{M}}$ is the fraction of the iron-containing minerals in the mantle.

The core mass fraction $X_{\mathrm{CMF}}$ of the planet mass $M_{\mathrm{p}}$ depends on the total iron content $X_{\mathrm{Fe}}$ and the iron that stays in the mantle:

$X_{\mathrm{CMF}} M_{\mathrm{p}}=X_{\mathrm{Fe}} M_{\mathrm{p}}-X_{\mathrm{FeM}}\left(1-X_{\mathrm{CMF}}\right) M_{\mathrm{p}}$,

and therefore:

$X_{\mathrm{CMF}}=\frac{X_{\mathrm{Fe}}-X_{\mathrm{FeM}}}{1-X_{\mathrm{FeM}}}$.

For our cases, the iron number in the mantle $\#_{F e}$ varies between 0 and 0.2 , which leads to iron mantle mass fractions $X_{\mathrm{FeM}}$ between 0 and 0.1457 . The planet's iron content varies between 0.15 and 0.80 as weight fractions, whereas the resulting core mass fraction $X_{\mathrm{CMF}}$ varies between 0.005 and 0.8 of the planet mass, depending on the amount of iron in the mantle.

\subsection{Core radius}

The core radius is also determined empirically via a scaling law, and depends on the core mass fraction and planet mass.

Even though the temperature inside the core only has a small effect of few percent on the core radius and on the overall planet radius, we differentiate here between our end-member temperature cases (the cold and the hot profile in Fig. 1), since our analytically derived scaling law for the core density below is based on the core radius, and a temperature-induced variation in the core radius. Hence, in the core, a temperature-independent density would scale up errors for the other planet parameters. We acknowledge, though, that for the further usage of the core radius after the core density calculation, the influence of the temperature dependence of the core radius is minor, and we 

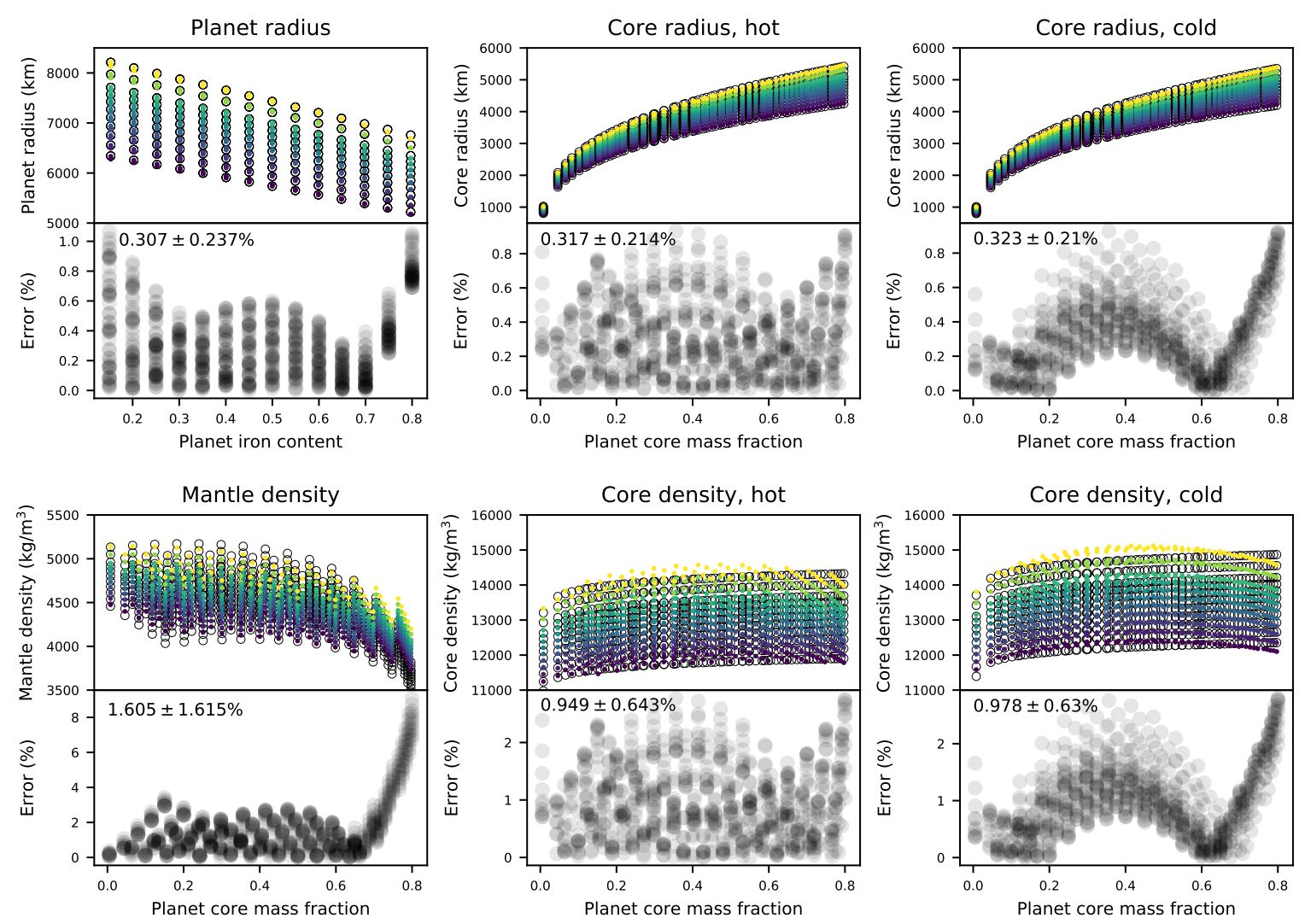

Fig. 4. Comparison between interior structure model data (filled circles) and predicted data from our parameterised model (black empty circles) for different planet parameters and planet masses, with colours ranging from 0.8 (dark blue) to 2 (yellow) Earth masses. Grey circles show the individual errors. The mean error with one standard deviation is listed for each error plot.

use the hot profile's core radius for all other calculations after Sect. 3.4:

$$
\begin{aligned}
& R_{\mathrm{c}, \text { hot }}[\mathrm{km}]=4850 X_{\mathrm{CMF}}^{0.328}\left(\frac{M_{\mathrm{p}}}{M_{\text {Earth }}}\right)^{0.266}, \\
& R_{\mathrm{c}, \text { cold }}[\mathrm{km}]=4790 X_{\mathrm{CMF}}^{0.328}\left(\frac{M_{\mathrm{p}}}{M_{\text {Earth }}}\right)^{0.266} .
\end{aligned}
$$

\subsection{Average density of the core}

The average core density is a direct result of the planet mass and core radius and can be derived analytically. The density profile as well as the entire interior structure depend on the assumed temperature profile. We therefore differentiate again between the cold temperature case (adiabatic increase from upper mantle to planet centre) and a hot temperature case (adding a temperature jump at the CMB reflecting the temperature difference at the end of mantle solidification, Sect. 3.9). Depending on the core radius calculated above for either the hot or the cold temperature profile, we obtain

$\rho_{\mathrm{c}, \mathrm{av}}\left[\frac{\mathrm{kg}}{\mathrm{m}^{3}}\right]=\frac{X_{\mathrm{CMF}} M_{\mathrm{p}}}{4 / 3 \pi\left(R_{\mathrm{c}} \cdot 1000\right)^{3}}$.

The density of the core is higher when assuming the cold temperature profile (without a temperature increase along the $\mathrm{CMB}$ ). Applying Eq. (11) for $R_{\mathrm{c}, \text { cold }}$ gives a reasonable approximation with errors similar to those of the hot case.

The variation of planet radius and core radius depending on planet iron content and core mass fraction when assuming a pure iron core is shown in the top row of Fig. 4 for different masses. The planet parameters calculated from the interiorstructure model are shown with coloured circles, where the colour corresponds to the planet mass (from 0.8 Earth masses in dark blue to 2 Earth masses in yellow). In addition, the predicted values for the same parameter cases are added with black, open circles. Figures 4-7 show additional comparisons between data and predicted values for all planet parameters and empirical scaling laws for thermodynamics parameters as derived in the next subsections.

\subsection{Average density of the mantle}

The average density of the mantle follows directly from the coremass fraction, the planet mass, and the core and planet radius. Here, we used the core radius, which was empirically given above for the hot profile, only, since our density error is not strongly affected by the core temperature variation, hence from now on $R_{\mathrm{c}}=R_{\mathrm{c}, \text { hot }}$. The errors with respect to our original interior structure data are given as combined error for both the hot and cold temperature profiles:

$\rho_{\mathrm{m}, \mathrm{av}}\left[\frac{\mathrm{kg}}{\mathrm{m}^{3}}\right]=\frac{\left(1-X_{\mathrm{CMF}}\right) M_{\mathrm{p}}}{4 / 3 \pi\left(R_{\mathrm{p}}^{3}-R_{\mathrm{c}}^{3}\right) \cdot 1000^{3}}$.

\subsection{Gravitational acceleration}

Based on the average mantle density and the core and planet radius, the surface and $\mathrm{CMB}$ gravitational acceleration can be 

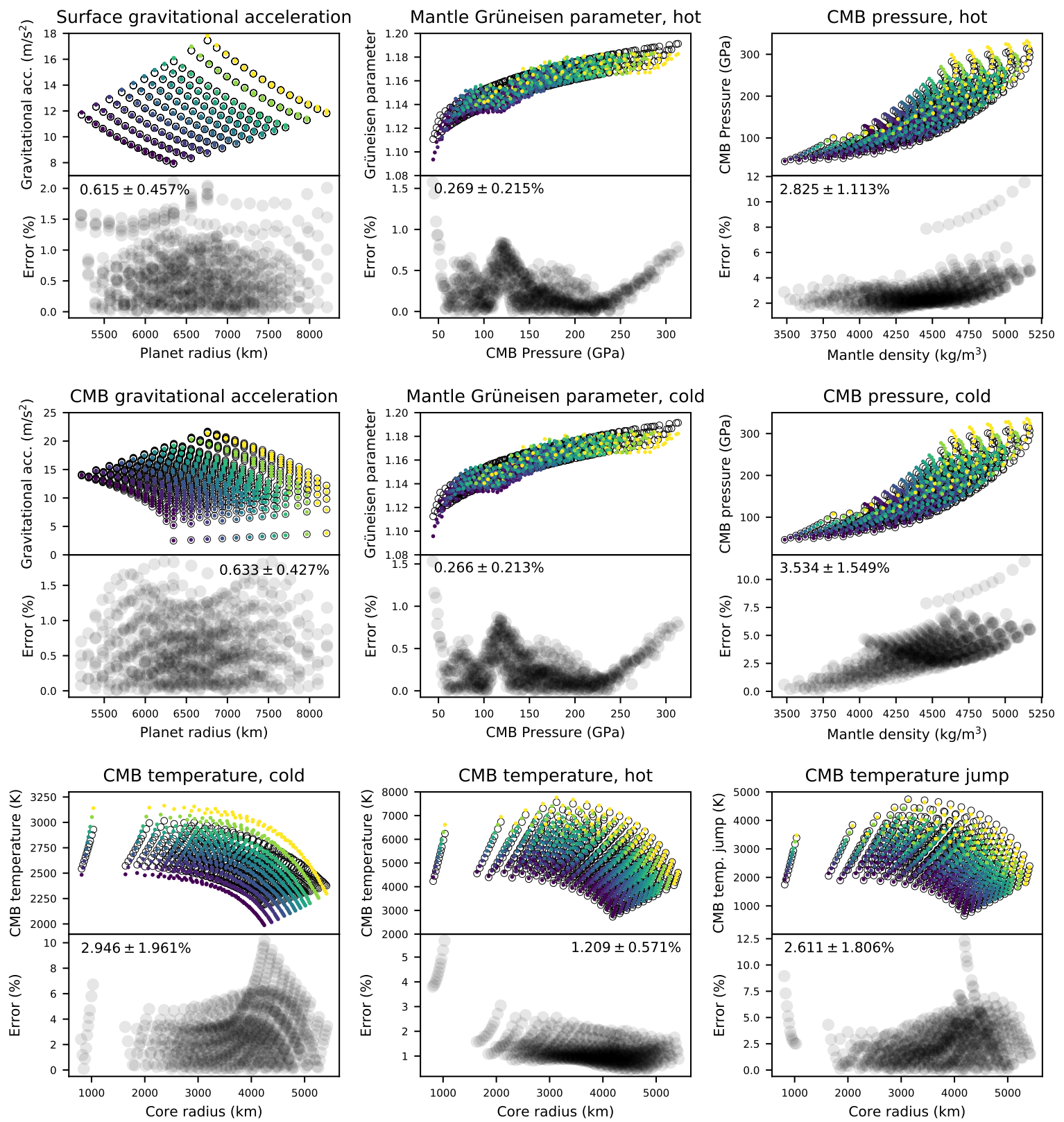

Fig. 5. Comparison between interior structure model data (filled circles) and parameterised model (black empty circles) for different planet parameters and planet masses. The mean error with 1 standard deviation is listed for each error plot. The colour coding follows that of Fig. 4.

calculated analytically as

$g_{0}\left[\frac{\mathrm{m}}{\mathrm{s}^{2}}\right]=\frac{G M_{\mathrm{p}}}{\left(R_{\mathrm{p}} \cdot 1000\right)^{2}}$

$g_{\mathrm{CMB}}\left[\frac{\mathrm{m}}{\mathrm{s}^{2}}\right]=\frac{G X_{\mathrm{CMF}} M_{\mathrm{p}}}{\left(R_{\mathrm{c}} \cdot 1000\right)^{2}}$,

for gravitational constant $G=6.67384 \times 10^{-11} \mathrm{~m}^{3} /\left(\mathrm{kg} \mathrm{s}^{2}\right)$.

The average mantle gravitational acceleration is then set as

$g_{\mathrm{m}, \mathrm{av}}=\frac{g_{0}+g_{\mathrm{CMB}}}{2}$.

The gravitational acceleration does not evolve linearly within the mantle (see Fig. 3), and an average of the surface and CMB values can only give a first approximation. The radially averaged gravitational acceleration from our interior structure model is, however, very close to this simplified parameterisation with errors of few percent, where the lowest errors (well below 1\%) occur for planets with a high iron content.

\subsection{Pressure at $C M B$}

As a first order approximation, in our parameterisation, the pressure at a specific depth can be calculated via the gravitational acceleration times the average mantle density times the depth of the mantle, hence

$p_{\mathrm{CMB}}[\mathrm{GPa}]=g_{\mathrm{m}, \mathrm{av}} \rho_{\mathrm{m}, \mathrm{av}}\left(R_{\mathrm{p}}-R_{\mathrm{c}}\right) \cdot 1000 \times 10^{-9}$.

In general, such a linear approximation is valid if the gravitational acceleration and the density do not change strongly with depth. In our range of investigated planetary masses and compositions, the approximation yields pressures comparable to the pressures that we obtain by integration from our interior structure model with an average error of 3\% (see Fig. 5). The largest 

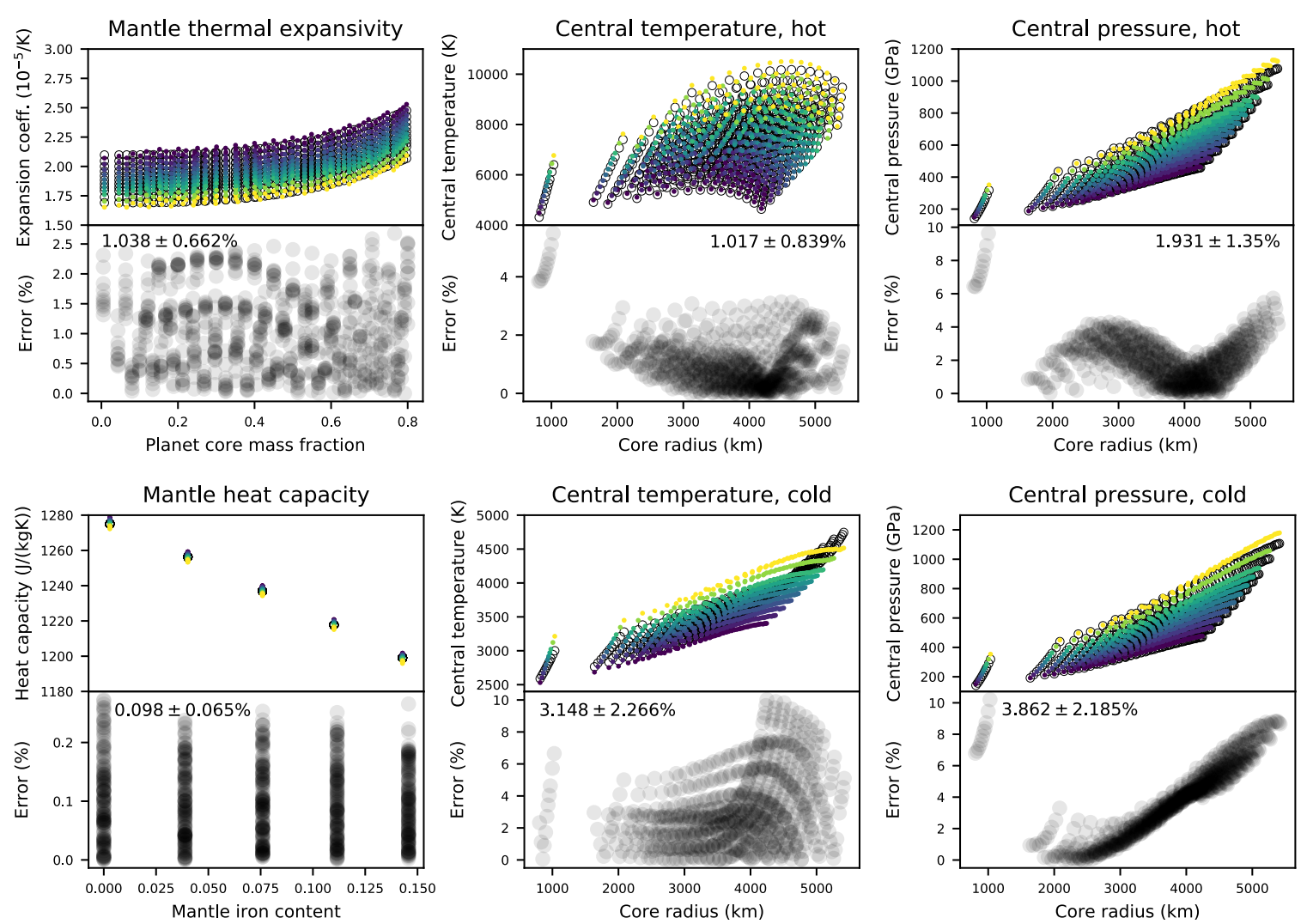

Fig. 6. Comparison between interior structure model data (filled circles) and parameterised model (black empty circles) for different planet parameters and planet masses. The mean error with 1 standard deviation is listed for each error plot. The colour coding follows that of Fig. 4.

errors appear for the more massive planets, where gravity and density more strongly depend on depth than in the case of Earthmass planets.

\subsection{Mantle properties}

The Grüneisen parameter is derived from the equations of state for the different mantle minerals (Stixrude \& Lithgow-Bertelloni 2011). For simplicity, we give here empirical scaling laws for average mantle values depending on the mantle depth (expressed here via the pressure at the CMB) and the mantle's iron content. The mantle scaling laws are derived from our interior structure model using the Birch-Murnaghan EoS (Stixrude \& Lithgow-Bertelloni 2011):

$\gamma_{\mathrm{m}, \mathrm{av}}=0.0356+0.96 \frac{p_{\mathrm{CMB}}^{0.03}}{\mathrm{GPa}}+0.14 X_{\mathrm{FeM}}^{1.16}$.

The average thermal expansion coefficient of the mantle is again derived from the equations of state by Stixrude \& Lithgow-Bertelloni (2011) and described here with an empirical scaling law depending on the planet mass and the thickness of the mantle, here expressed via the core-mass fraction,

$\alpha_{\mathrm{m}, \mathrm{av}}\left[\frac{1}{\mathrm{~K}}\right]=\left(13+0.738 X_{\mathrm{CMF}}-11\left(\frac{M_{\mathrm{p}}}{M_{\text {Earth }}}\right)^{0.04}\right) \times 10^{-5}$.

The average mantle heat capacity mostly depends on the composition of the mantle, and more specifically the iron content in the mantle, as

$C_{\mathrm{p}, \mathrm{m}, \mathrm{av}}\left[\frac{\mathrm{J}}{\mathrm{kg} \mathrm{K}}\right]=1275-585 X_{\mathrm{FeM}}^{1.06}$.

\subsection{Temperature increase at the $C M B$}

Based on the ideas formulated in Stixrude (2014), we assumed that the uppermost temperature of the core reflects the melting temperature of the mantle at the same pressure as a first-order approximation of temperatures in the core at the magma ocean freezing stage. We used the silicate post-perovskite melting temperature from the same study and took into account that iron reduces the melting temperature to obtain that

$T_{\mathrm{CMB}, \mathrm{hot}}[\mathrm{K}]=\frac{5400\left(\frac{p_{\mathrm{CMB}, \mathrm{hot}}}{140 \mathrm{GPa}}\right)^{0.48}}{1-\ln \left(1-\# \mathrm{Fe}_{\mathrm{M}}\right)}$

The CMB temperature for the warm case would then be (Stixrude 2014):

$T_{\mathrm{CMB}, w a r m}[\mathrm{~K}]=\frac{5400\left(\frac{p_{\mathrm{CMB}, \mathrm{hot}}}{140 \mathrm{GPa}}\right)^{0.48}}{1-\ln \left(1-X_{\mathrm{M} 0}-\# \mathrm{Fe}_{\mathrm{M}}\right)}$,

where $X_{\mathrm{M} 0}=0.11$ is a factor accounting for the deviation from the liquidus temperature due to other minerals present in the mantle (similarly to the effect that iron has on reducing the melting temperature), and which was fitted to Earth's mantle for an iron number of $\# \mathrm{Fe}_{\mathrm{M}}=0.1$.

On the other hand, we needed to determine the adiabatic mantle temperature and how it extends towards the core-mantle boundary. Since the mantle temperature does not vary in our two temperature scenarios, the lower mantle temperature is similar 

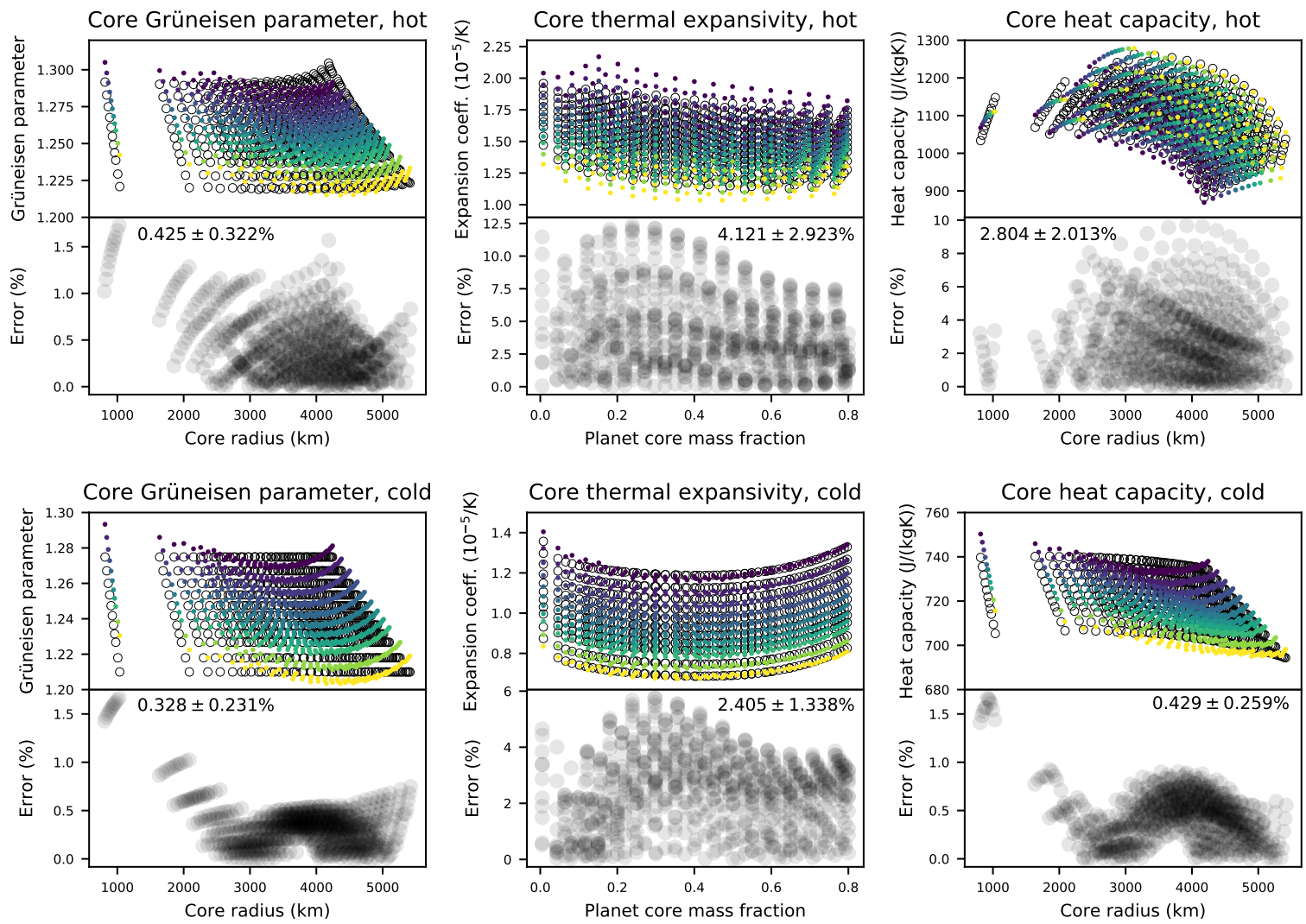

Fig. 7. Comparison between interior structure model data (filled circles) and parameterised model (black empty circles) for different planet parameters and planet masses. The mean error with one standard deviation is listed for each error plot. The colour coding follows that of Fig. 4.

to the $\mathrm{CMB}$ temperature in the cold case (neglecting the slight change in core radius from hot to cold core scenario). Equally, we kept the other mantle parameters as derived for the hot case scenario, since the change in core radius for different core temperatures has only a small influence on the gravitational acceleration at the $\mathrm{CMB}$ and other parameters derived from $R_{\mathrm{c}}$.

$T_{\mathrm{CMB}, \text { cold }}[\mathrm{K}]=T_{\mathrm{um}} \cdot \exp \left(d_{\mathrm{T}} \frac{g_{\mathrm{m}, \mathrm{av}} \alpha_{\mathrm{m}, \mathrm{av}}}{C_{\mathrm{p}, \mathrm{m}, \mathrm{av}}}\left(R_{\mathrm{p}}-R_{\mathrm{c}, \mathrm{hot}}-D_{\mathrm{l}}\right) \cdot 1000\right)$,

where $T_{\text {um }}$ is the upper-mantle temperature (here we assume $2000 \mathrm{~K}$ for simplicity) at the bottom of the lithosphere $D_{1}=250 \mathrm{~km}$. The adiabatic temperature profile is not linear over the mantle. Instead, the adiabatic temperature increase is stronger in the upper mantle and more reduced for higher pressures. Using mantle-averaged parameters in Eq. (22) as needed in our parameterisation would overestimate the CMB temperature. We therefore introduced a pre-factor $d_{\mathrm{T}}$ that we empirically derived to obtain the best approximation of the CMB temperature in comparison to our integrated temperature profile in the interior structure model. Within our modelled mass and composition range, we find that $d_{\mathrm{T}} \approx 0.5$.

The temperature jump can be calculated from the difference between the hot CMB temperature and the cold temperature profile. To account for the existence of a possible $\mathrm{D}^{\prime \prime}$ layer and to get a better estimate of the temperature jump from our interior structure model, we calculated the temperature jump as difference between the hot CMB temperature and the adiabatic mantle temperature $500 \mathrm{~km}$ above the CMB (the here assumed thickness of the $\mathrm{D}^{\prime \prime}$ layer) $T_{\mathrm{D}^{\prime \prime}}$ :
$T_{\mathrm{D}^{\prime \prime}}[\mathrm{K}]=T_{\mathrm{um}} \cdot \exp \left(d_{\mathrm{T}} \frac{g_{\mathrm{m}, \mathrm{av}} \alpha_{\mathrm{m}, \mathrm{av}}}{C_{\mathrm{p}, \mathrm{m}, \mathrm{av}}}\left(R_{\mathrm{p}}-R_{\mathrm{c}, \mathrm{hot}}-D_{\mathrm{l}}-D^{\prime \prime}\right) \cdot 1000\right)$,

and finally,

$\Delta T[\mathrm{~K}]=T_{\mathrm{CMB}, \mathrm{hot}}-T_{\mathrm{D}^{\prime \prime}}$.

\subsection{Core properties}

Based on the core temperature determination, we can now derive estimates for the core thermodynamic properties, namely the Grüneisen parameter, the thermal expansion coefficient and the heat capacity. The scaling laws were first derived for the cold temperature profile (no temperature jump across the $\mathrm{D}^{\prime \prime}$ layer) and then adapted to include the dependence of the temperature contrast. These formulations then also allow application to other (e.g. intermediate) temperature profile cases such as the "warm" case shown in Fig. 1. The influence of core temperature on average core properties is shown in Fig. 7. We obtain the following empirical relationships for the main averaged core properties derived from our interior structure model using the Holzapfel EoS (Bouchet et al. 2013):

$$
\begin{gathered}
\gamma_{\mathrm{c}, \mathrm{av}}=\left(1.66-0.4 \cdot\left(\frac{M_{\mathrm{p}}}{M_{\text {Earth }}}\right)^{0.17}\right) \cdot\left(1+\Delta T^{-0.58}\right), \\
\alpha_{\mathrm{c}, \mathrm{av}}\left[\frac{1}{\mathrm{~K}}\right]=\left(6.33+0.484 X_{\mathrm{CMF}}^{4.464}-0.0462 \rho_{\mathrm{c}, \mathrm{av}, \mathrm{cold}}^{0.509}\right) \\
\times\left(1+0.0034 \Delta T^{0.6524}\right) \times 10^{-5},
\end{gathered}
$$



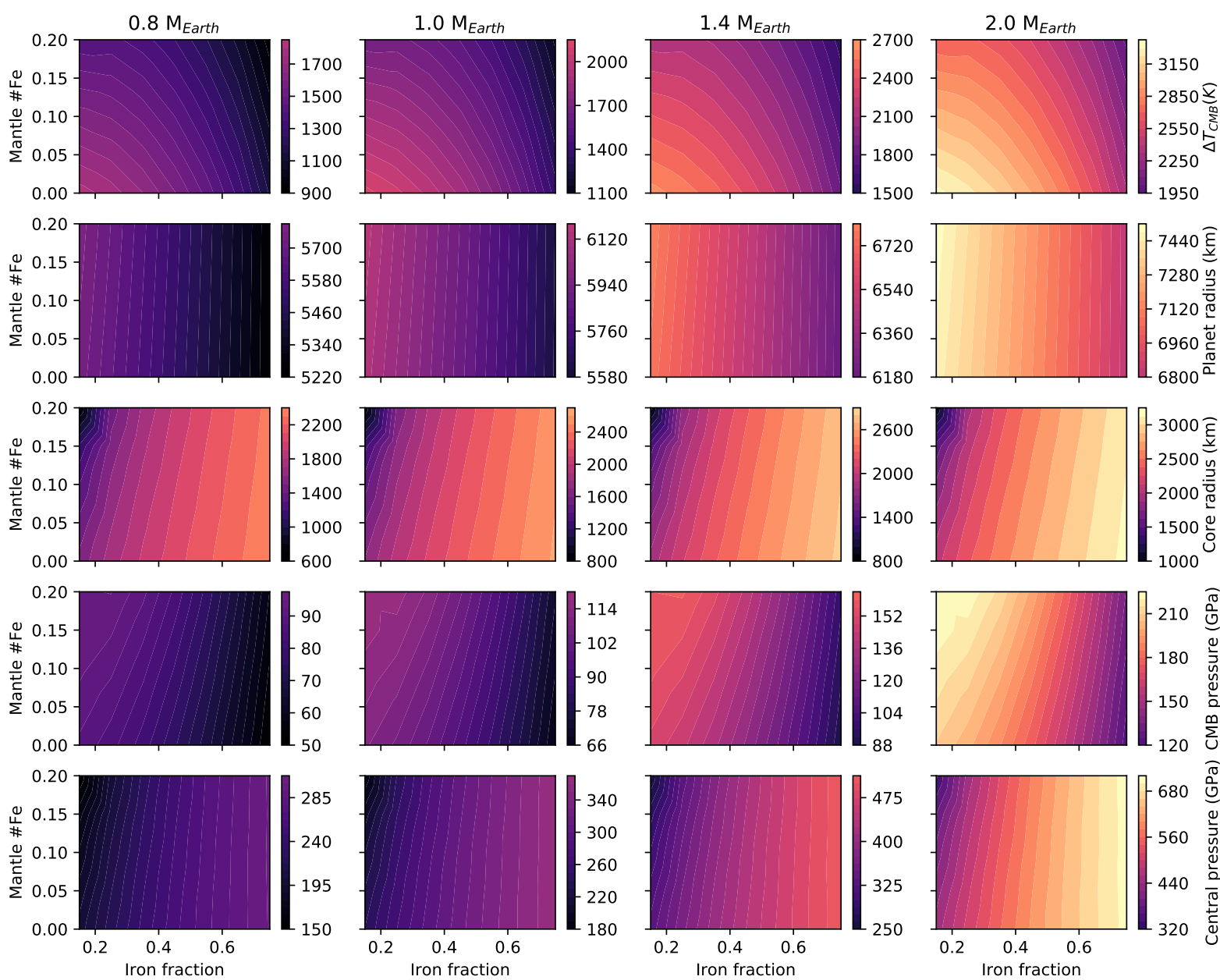

Fig. 8. Main planetary parameters for the hot end-member case depending on planet mass (different columns), planet iron content, and iron number in mantle, assuming an $\mathrm{Mg}-\mathrm{Si}-\mathrm{O}$ ratio similar to Earth. Each row shows a different planet parameter: temperature jump at core-mantle boundary, planet radius, core radius, and pressure at the core-mantle boundary and in the centre of the planet. Each row uses a fixed colour scale to highlight the variation with planet mass.

$$
\begin{aligned}
C_{\mathrm{p}, \mathrm{c}, \mathrm{av}}\left[\frac{\mathrm{J}}{\mathrm{kg} \mathrm{K}}\right]= & \left(880+\left(0.0163 T_{\mathrm{CMB}, \text { cold }}-191\right)\left(\frac{M_{\mathrm{p}}}{M_{\text {Earth }}}\right)^{0.286}\right) \\
& \times\left(1+0.0023 \Delta T^{0.69}\right) .
\end{aligned}
$$

\subsection{Centre-of-planet values}

Based on the core-averaged thermodynamic parameters and radii calculations above, we can derive the pressure and temperature at the centre of the planet via

$$
p_{\text {centre }}[\mathrm{GPa}]=p_{\mathrm{CMB}}+d_{\mathrm{p}} g_{\mathrm{CMB}} \rho_{\mathrm{c}, \mathrm{av}} R_{\mathrm{c}} \cdot 1000 \times 10^{-9} \text {. }
$$

As before, $d_{\mathrm{p}}$ is a pre-factor that we introduce to obtain the best approximation of the central pressure in our parameterised model compared to the interior structure model. The average gravitational acceleration in the core can be obtained by applying approximately half the CMB value. The density in the core increases with increasing pressure, and the pressure increase is not linear with depth. Comparison with our interior structure model reveals that a factor of $d_{\mathrm{p}} \approx 0.58$ yields the lowest error in a central pressure estimate. For the cold temperature profile, we apply the cold estimates for CMB pressure, average core density, and core radius:

$T_{\text {centre }}[\mathrm{K}]=T_{\mathrm{CMB}} \cdot \exp \left(d_{\mathrm{T}} \frac{g_{\mathrm{CMB}} \alpha_{\mathrm{c}, \mathrm{av}}}{C_{\mathrm{p}, \mathrm{c}, \mathrm{av}}} R_{\mathrm{c}}\right)$.

Similarly, for the cold temperature profile, $T_{\mathrm{CMB}}, R_{\mathrm{c}, \text { cold }}$, $\alpha_{\mathrm{c}, \mathrm{av}, \mathrm{cold}}$, and $C_{\mathrm{p}, \mathrm{c}, \mathrm{av}, \text { cold }}$ need to be applied.

Our parameterisations build upon one another - for example, to calculate the temperature in the centre of the planet, we first need to derive the CMB temperature and the gravitational acceleration at the $\mathrm{CMB}$, core thermodynamic parameters as well as the core radius. Still, as can be seen in Figs. 4-7, our errors stay within few percent for all scaling laws, since we mainly used first-principle relationships to relate the different planet properties to each other.

Figures 8 and 9 give a more quantitative overview of how the main planet parameters (radii, pressures, and temperature jump at the CMB) as well as core parameters (temperature, density, heat capacity, thermal expansion coefficient, and Grüneisen parameter) change with planet mass, iron fraction and mantle iron number.

\section{Discussion}

We derive scaling relationships for the most important planet parameters and thermodynamic parameters in mantle and core for planets composed of $\mathrm{Mg}, \mathrm{Si}, \mathrm{Fe}$, and $\mathrm{O}$, which are the main elements the Earth is made of. Though other chemical elements (for example $\mathrm{Na}, \mathrm{Ca}$, and $\mathrm{Al}$ ) and minerals (such as pyroxenes, $\mathrm{SiO}_{2}, \mathrm{Ca}$-perovskite) are important for Earth's crustal

page 10 of 13 

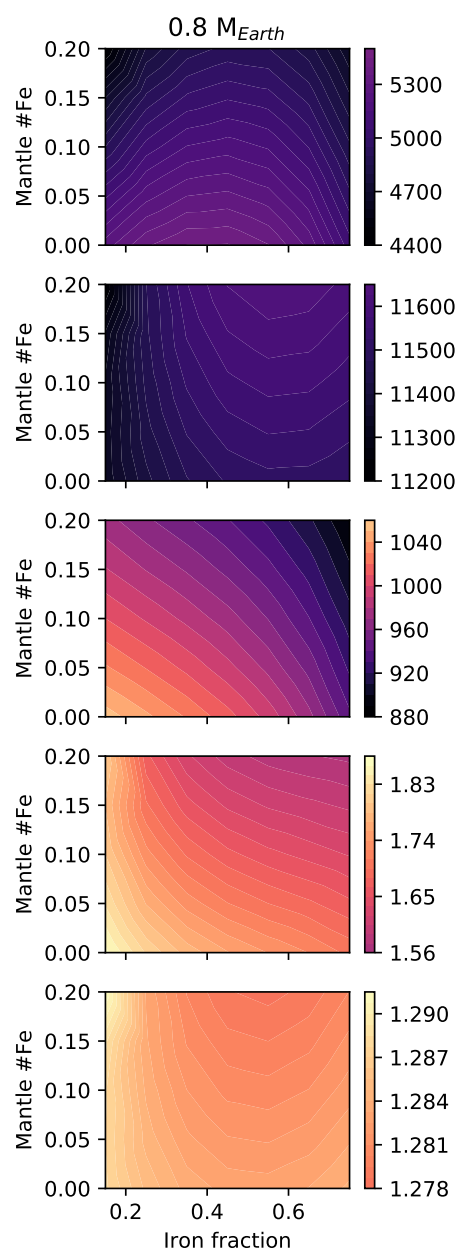

$1.0 \mathrm{M}_{\text {Earth }}$
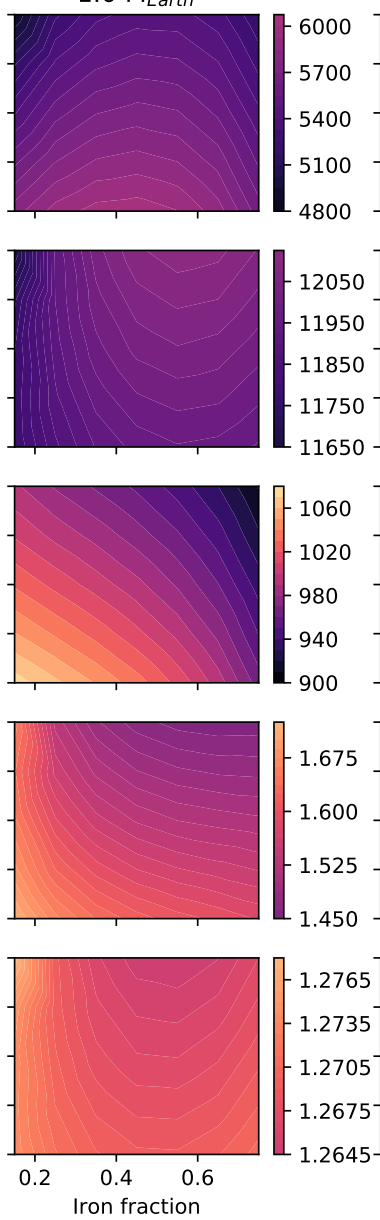

$1.4 M_{\text {Earth }}$
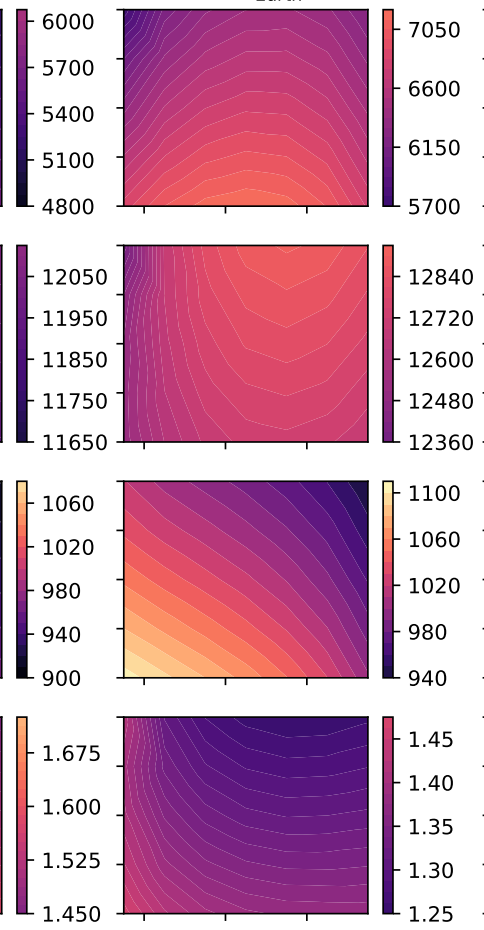
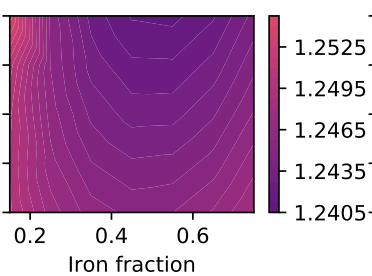

$2.0 \mathrm{M}_{\text {Earth }}$
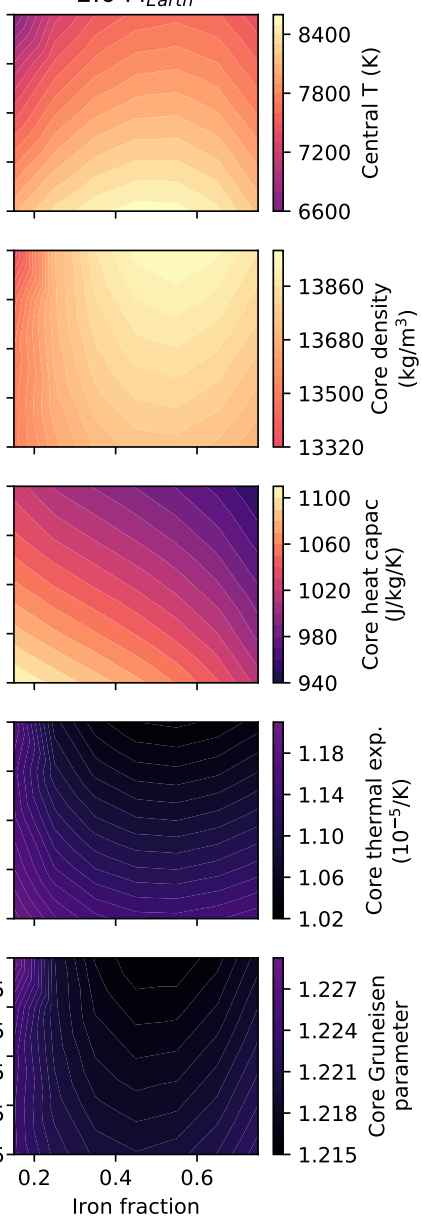

Fig. 9. Main thermodynamic parameters in the core for the hot end-member case depending on planet mass (different columns), planet iron content and iron number in mantle, assuming an $\mathrm{Mg}-\mathrm{Si}-\mathrm{O}$ ratio similar to Earth. First row: temperature in the centre of the planet, followed by the averaged core thermodynamic values for density, heat capacity, thermal expansion coefficient, and Grüneisen parameter. Each row uses a fixed colour scale to highlight the variation with planet mass.

composition and chemical processes in the mantle (e.g. for the hydration of mantle minerals, partitioning behaviour upon melting), the interior structure and main thermodynamic properties are mostly defined by olivine and its high-pressure equivalents, perovskite, post-perovskite, magnesiowustite, and iron. We therefore limit our study to these main components. Since $\mathrm{Mg}, \mathrm{Fe}$, and $\mathrm{Si}$ condense at similar temperatures in the accretion disk (Lodders 2003), we expect that for exoplanets forming in the inner part of the accretion disk, these elements are the major players for defining the mantle minerals of rocky planets (equally to the Solar System). Here, we therefore investigated only planets with a pure iron core and a rocky mantle made of $\mathrm{Mg}_{2} \mathrm{SiO}_{4}$ and $\mathrm{Fe}_{2} \mathrm{SiO}_{4}$ in the upper mantle, and $\mathrm{MgO}, \mathrm{FeO}$, $\mathrm{MgSiO}_{3}$, and $\mathrm{FeSiO}_{3}$ in the lower mantle, to infer the variations in basic planetary parameters depending on composition, mass, and temperature profile.

It has been suggested that close-in exoplanets may contain higher $\mathrm{Al}$ and $\mathrm{Ca}$ abundances than observed in the Solar System (Dorn et al. 2019) and that stars with very high carbon abundances may lead to planets with completely different chemistries (Kuchner \& Seager 2005; Unterborn et al. 2014; Nisr et al. 2017; Madhusudhan et al. 2012). However, it is not unlikely to assume that the majority of rocky exoplanets formed in close proximity to their star (as was the case for the planets in the inner Solar System) would show mantle minerals quite similar to Earth with variations in $\mathrm{Mg}-\mathrm{Fe}-\mathrm{Si}$ concentrations depending on the stellar evolution history (Bitsch \& Battistini 2020).

Dorn et al. (2015) and other recent publications have also shown that the ratio of these elements is related to the abundances of $\mathrm{Mg}, \mathrm{Si}$ and $\mathrm{Fe}$ in the star and can in some cases be observed in the stellar spectrum. A higher or lower Fe content in the stellar spectrum, for example, would suggest larger or smaller iron cores in rocky planets orbiting that star, though other processes such as impacts can change the core-to-mantle ratio (as seen for the Moon and Mercury in the Solar System). Also, the chemistry in the magma ocean (specifically the redox state) influences the differentiation of iron into the core (Elkins-Tanton \& Seager 2008; Wohlers \& Wood 2017). It is therefore useful to have a simple tool, as provided here, to estimate the first-order influence of variable iron contents in the planet as well as the distribution of iron between mantle and core.

Our parameterisations are derived from interior-structure models of planets with a limited mass range ( 0.8 to 2 Earth masses), since the equations of state that we use were derived for the Earth's pressure range. Scaling the equations of state to more massive planets adds a potential error due to a lack of experimental or ab initio data. In addition, we do not yet fully understand the expected mineralogy and rheology at pressures well beyond 


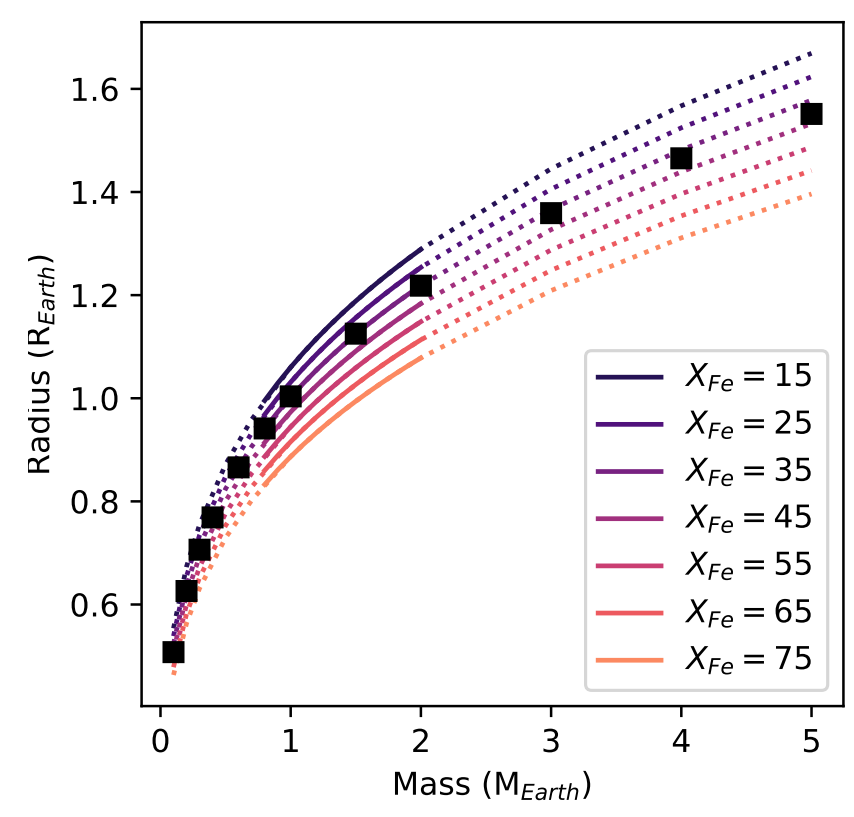

Fig. 10. Mass-radius relationship as predicted by our scaling laws for variable iron contents but with a fixed mantle iron number of 0.1 . Solid lines refer to the range of masses over which our parameterisations were derived (i.e. close to Earth pressures and temperatures, 0.8 to 2 Earth masses) and dotted lines refer to an extrapolation of our scaling laws beyond that range. Black boxes show, for comparison, the actual radius and mass values calculated with our interior structure model for an iron content of 0.35 and for masses from 0.1 to 5 Earth masses.

several hundred GPa, and different regimes (such as a different rheology regime, Karato 2011, or post-perovskite dissociation, Umemoto \& Wentzcovitch 2011) have been proposed. In the investigated mass range, our parameterisations are valid with the errors identified for each scaling law. Nevertheless, due to the form of our scaling laws, which are mostly derived from firstorder principles, our parameterisations can be extrapolated up to approximately five Earth masses, which can be seen in Fig. 10. They are not, however, valid for a planet with a different composition, meaning a planet that is not mainly composed of Fe, $\mathrm{Mg}, \mathrm{Si}$, and $\mathrm{O}$, or that contains mainly $\mathrm{SiO}_{2}$ instead of $\mathrm{Mg}$ - and Fe-rich silicates.

A major factor for determining the size of the metal core is the efficiency of differentiation between mantle and core, mostly during the accretion and magma ocean stage of a planet, when iron droplets can form and sink through the mantle towards the gravitational centre of the planet, hence the core. This process also depends on the chemistry of the mantle, since the iron would be assumed to be in equilibrium with the magma ocean. If the latter is oxidised, then the iron would form iron oxides instead of droplets of metallic iron, and would float instead of sinking to form a core (e.g. Elkins-Tanton \& Seager 2008). There are several factors that influence the oxidation stage of the mantle (such as delivery of oxidised material, loss of reduced species to the atmosphere, formation of the core from reduced material, and disproportionation of perovskite in the lower mantle, Gaillard et al. 2020; Frost \& McCammon 2008). It would go beyond the scope of this study to model variations in magma ocean oxidation states. Instead, we varied the amount of iron that may stay trapped in the mantle, which is the effect that an oxidised mantle has on the interior structure. Similarly, lighter elements would be expected to accumulate in the core during core differentiation. We still do not know which light elements and in what abundance they exist in Earth's core (several elements have been suggested such as $\mathrm{S}, \mathrm{O}, \mathrm{C}, \mathrm{H}, \mathrm{Si}$, and $\mathrm{Mg}$, Hirose et al. 2013; Badro et al. 2018). Equations of states describing the thermodynamic behaviour of iron alloys with these lighter elements are, however, sparse for pressures exceeding Earth's core pressures. In this study, we therefore only concentrated on pure iron cores without impurities.

The temperature profile that we used for deriving the interior structure model and the thermodynamic parameters for mantle and core were derived from a hot to a cold case to encompass a large range of temperature variations in the core, which would reflect the thermal evolution and cooling of the core over time. For simplicity, here the mantle temperature was taken as constant, since the change in mantle temperature is expected to be about an order of magnitude smaller than for the core with limited effects in the interior structure of mantle properties (Herzberg et al. 2010). The profile that we assumed here for the mantle is an adiabatic profile that is correct for a strongly convecting mantle. A different profile might be expected if the lower mantle were rather sluggish, as suggested by Stamenković et al. (2012), Noack \& Breuer (2014), Tackley et al. (2013) for rocky planets more massive than Earth (super-Earths), or if the magma ocean crystallised not from the bottom up but in a different way (e.g. Nomura et al. 2011), or if a mantle overturn occurred after magma ocean crystallisation (Elkins-Tanton 2008; Plesa et al. 2014). However, for the latter two cases, temperature variations would soon homogenise towards an adiabatic profile due to mantle convection. On the other hand, a possible sluggish lower mantle for massive super-Earths might evolve into a self-regulating state to allow for more rapid cooling of the lower mantle, leading again to a temperature profile close to what we have assumed here (Tackley et al. 2013). In any case, our assumed hot temperature scenario exceeds previous estimates on lower mantle temperatures in super-Earths by $1000 \mathrm{~s}$ of Kelvin (see Fig. 3), which would trigger fast convection in the entire mantle due to hot plumes forming at the $\mathrm{CMB}$, thus again allowing for an adiabatic mantle temperature profile as assumed.

The reduction in planet radius by thermal contraction from our hot end-member case to the cold end-member case is negligible, and we can therefore derive one scaling law for the planet radius independent of temperature (Eq. (5)), with an error of only $0.307 \%$ over all investigated planet structure models. However, this may also be related to our assumption of a fixed mantle temperature profile. If we consider the variations in density that we would expect if part of or all of the mantle were molten, then a larger variation in planet radius with temperature would be expected (Bower et al. 2019). The temperature also has a small effect on the core radius (Eqs. (9) and (10)), with a change of $1.2 \%$ on average from one end-member scenario to the other. Though this is still negligibly small, the change in core radius already affects the accuracy of the core scaling laws that build up on the core radius (such as core density and pressure at the $\mathrm{CMB}$ ). The temperature in the core has a huge effect on other thermodynamic parameters, such as the thermal expansion coefficient and heat capacity (see Fig. 3), which are critical to understanding the thermal profile and cooling behaviour of the core, as well as its dynamics possibly leading to a magnetic field.

In general, the effect of temperature on the interior structure can be derived from the temperature-sensitivity of the core and mantle densities. In a simple approximation, the density of a material depends on temperature, following

$\rho(T)=\rho\left(T_{\text {ref }}\right) \cdot \exp \left(-\alpha\left(T-T_{\text {ref }}\right)\right)$ 
where $\alpha$ is again the thermal expansion coefficient and $T_{\text {ref }}$ the reference temperature for which the density was derived. In the results section, we show how the thermal expansion coefficient depends on composition and temperature. Assuming here a thermal expansion coefficient of $2 \times 10^{-5} 1 / \mathrm{K}$ for mantle and core for simplicity (corresponding to a hot core scenario), we can estimate that for an increase in average planet temperature of $1000 \mathrm{~K}$, the total planet density would be $5 \%$ lower, leading to an increase in planet radius of $1.7 \%$. This does not take into account additional density variations due to formation of melt, which would have a larger influence on planet radius (Bower et al. 2019). It should also be noted that the effect of temperature on planet radius is more extreme when large amounts of water (possibly in the state of higher pressure ice) are present, and it can exceed several percent (Noack et al. 2016).

\section{Conclusions}

We provide the community with robust scaling laws for the interior structure, temperature profiles, and core- and mantleaveraged thermodynamic properties for planets composed of Earth's main minerals, but with variable compositions of iron and silicates. Our investigated temperature profiles reflect the thermal evolution of the core from a hot profile after magma ocean solidification to a cold state after efficient cooling of the planet. The initial temperatures derived in our study exceed previous estimates on mantle temperatures for more massive planets, and studies modelling interior dynamics based on such temperature estimates should be re-investigated. The scaling laws make it possible to mimic variations in core thermodynamic properties over time (i.e. due to cooling of the core) in a multitude of applications, for example for deriving composition- and temperature-dependent mass radius scaling laws, for estimating the solidification of a core, or for determining possible magnetic field evolution inside rocky exoplanets' cores.

Acknowledgements. We would like to thank the editor and Craig O'Neill for their suggestions that helped to improve the manuscript. ML was funded by the European Union's Horizon 2020 research and innovation program under the Marie Skłodowska-Curie Grant Agreement No. 795289. The authors appreciate the support of ELSI, Tokyo, to host the Planetary Diversity Workshop in 2016, and which initiated this study. ML acknowledges funding of a COST Short Term Stay Mission from the COST Action TD 1308 ORIGINS. The interior structure profiles, an online tool and the link to the repository including all Python Jupyter notebooks, that were needed to derive the scaling laws and figures of this study, as well as an implementation of the scaling laws in Python are available on the following website: http://geodyn-chic.de/tools. We would like to thank Alexander Balduin for setting up this website.

\section{References}

Abe, Y. 1997, Phys. Earth Planet. Inter., 100, 27

Badro, J., Aubert, J., Hirose, K., et al. 2018, Geophys. Res. Lett., 45, 13

Ballmer, M. D., Lourenço, D. L., Hirose, K., Caracas, R., \& Nomura, R. 2017, Geochem. Geophys. Geosyst., 18, 2785

Baumeister, P., MacKenzie, J., Tosi, N., \& Godolt, M. 2018, European Planetary Science Congress 2018

Bitsch, B., \& Battistini, C. 2020, A\&A, 633, A10

Bouchet, J., Mazevet, S., Morard, G., Guyot, F., \& Musella, R. 2013, Phys. Rev. B, 87, 094102
Boukaré, C.-E., Parmentier, E., \& Parman, S. 2018, Earth Planet. Sci. Lett., 491, 216

Bower, D. J., Kitzmann, D., Wolf, A. S., et al. 2019, A\&A, 631, A103

Connolly, J. 2009, Geochem. Geophys. Geosyst., 10, Q10014

Crowley, J. W., \& O’Connell, R. J. 2012, Geophys. J. Int., 188, 61

Dorn, C., Khan, A., Heng, K., et al. 2015, A\&A, 577, A83

Dorn, C., Noack, L., \& Rozel, A. 2018, A\&A, 614, A18

Dorn, C., Harrison, J. H., Bonsor, A., \& Hands, T. O. 2019, MNRAS, 484, 712

Durek, J. J., \& Ekström, G. 1996, Bull. Seismol. Soc. Am., 86, 144

Elkins-Tanton, L. T. 2008, Earth Planet. Sci. Lett., 271, 181

Elkins-Tanton, L. T., \& Seager, S. 2008, ApJ, 688, 628

Frost, D., \& McCammon, C. 2008, Annu. Rev. Earth Planet. Sci., 36, 389

Gaillard, F., Bouhifd, M., Furi, E., et al. 2020, Space Sci. Rev., submitted

Grasset, O., Schneider, J., \& Sotin, C. 2009, ApJ, 693, 722

Hakim, K., Rivoldini, A., Van Hoolst, T., et al. 2018, Icarus, 313, 61

Herzberg, C., Condie, K., \& Korenaga, J. 2010, Earth Planet. Sci. Lett., 292, 79

Hirose, K., Labrosse, S., \& Hernlund, J. 2013, Annu. Rev. Earth Planet. Sci., 41, 657

Karato, S.-I. 2011, Icarus, 212, 14

Kuchner, M. J., \& Seager, S. 2005, ArXiv e-prints [arXiv:astro-ph/0504214]

Labrosse, S., Hernlund, J., \& Coltice, N. 2007, Nature, 450, 866

Lodders, K. 2003, ApJ, 591, 1220

Madhusudhan, N., Lee, K. K., \& Mousis, O. 2012, ApJ, 759, L40

Maurice, M., Tosi, N., Samuel, H., et al. 2017, J. Geophys. Res.: Planets, 122, 577

McDonough, W. F., \& Sun, S.-S. 1995, Chem. Geol., 120, 223

Nakajima, M., \& Stevenson, D. J. 2015, Earth Planet. Sci. Lett., 427, 286

Nisr, C., Meng, Y., MacDowell, A., et al. 2017, J. Geophys. Res.: Planets, 122, 124

Noack, L., \& Breuer, D. 2014, Planet. Space Sci., 98, 41

Noack, L., Godolt, M., von Paris, P., et al. 2014, Planet. Space Sci., 98, 14

Noack, L., Höning, D., Rivoldini, A., et al. 2016, Icarus, 277, 215

Noack, L., Rivoldini, A., \& Van Hoolst, T. 2017, Phys. Earth Planet. Inter., 269, 40

Nomura, R., Ozawa, H., Tateno, S., et al. 2011, Nature, 473, 199

O’Neill, C., Lenardic, A., Weller, M., et al. 2016, Phys. Earth Planet. Inter., 255, 80

Papuc, A. M., \& Davies, G. F. 2008, Icarus, 195, 447

Plesa, A.-C., Tosi, N., \& Breuer, D. 2014, Earth Planet. Sci. Lett., 403, 225

Rogers, L., \& Seager, S. 2010, ApJ, 712, 974

Sandu, C., Lenardic, A., O’Neill, C., \& Cooper, C. 2011, Int. Geol. Rev., 53, 1392

Stamenković, V., \& Breuer, D. 2014, Icarus, 234, 174

Stamenković, V., Breuer, D., \& Spohn, T. 2011, Icarus, 216, 572

Stamenković, V., Noack, L., Breuer, D., \& Spohn, T. 2012, ApJ, 748, 41

Stein, C., Schmalzl, J., \& Hansen, U. 2004, Phys. Earth Planet. Inter., 142, 225

Stevenson, D. J., Spohn, T., \& Schubert, G. 1983, Icarus, 54, 466

Stixrude, L. 2014, Philos. Trans. R. Soc. A: Math. Phys. Eng. Sci., 372, 20130076

Stixrude, L., \& Lithgow-Bertelloni, C. 2011, Geophys. J. Int., 184, 1180

Tackley, P. J., Ammann, M., Brodholt, J. P., Dobson, D. P., \& Valencia, D. 2013, Icarus, 225, 50

Thomas, P., Tajeddine, R., Tiscareno, M., et al. 2016, Icarus, 264, 37

Umemoto, K., \& Wentzcovitch, R. M. 2011, Earth Planet. Sci. Lett., 311, 225

Unterborn, C. T., \& Panero, W. R. 2017, ApJ, 845, 61

Unterborn, C. T., Kabbes, J. E., Pigott, J. S., Reaman, D. M., \& Panero, W. R. 2014, ApJ, 793, 124

Van Heck, H., \& Tackley, P. 2008, Geophys. Res. Lett., 35, L19312

Van Hoolst, T., Noack, L., \& Rivoldini, A. 2019, Adv. Phys.: X, 4, 1630316

Valencia, D., O'Connell, R. J., \& Sasselov, D. 2006, Icarus, 181, 545

Valencia, D., O'Connell, R. J., \& Sasselov, D. D. 2007, ApJ, 670, L45

Verhoeven, O., Rivoldini, A., Vacher, P., et al. 2005, J. Geophys. Res.: Planets, 110, E04009

Wagner, F., Sohl, F., Hussmann, H., Grott, M., \& Rauer, H. 2011, Icarus, 214, 366

Wohlers, A., \& Wood, B. J. 2017, Geochim. Cosmochim. Acta, 205, 226

Zhang, T., Baumjohann, W., Russell, C., Luhmann, J., \& Xiao, S. 2016, Sci. Rep., 6, 23537 